\title{
Artificial intelligence-supported lung cancer detection by multi-institutional readers with multi-vendor chest radiographs: a retrospective clinical validation study
}

Daiju Ueda ${ }^{1 *}$ (D), Akira Yamamoto', Akitoshi Shimazaki', Shannon Leigh Walston', Toshimasa Matsumoto', Nobuhiro Izumi ${ }^{2}$, Takuma Tsukioka², Hiroaki Komatsu², Hidetoshi Inoue ${ }^{2}$, Daijiro Kabata ${ }^{3}$, Noritoshi Nishiyama ${ }^{2}$ and Yukio Miki ${ }^{1}$

\begin{abstract}
Background: We investigated the performance improvement of physicians with varying levels of chest radiology experience when using a commercially available artificial intelligence (Al)-based computer-assisted detection (CAD) software to detect lung cancer nodules on chest radiographs from multiple vendors.

Methods: Chest radiographs and their corresponding chest $C T$ were retrospectively collected from one institution between July 2017 and June 2018. Two author radiologists annotated pathologically proven lung cancer nodules on the chest radiographs while referencing $C T$. Eighteen readers (nine general physicians and nine radiologists) from nine institutions interpreted the chest radiographs. The readers interpreted the radiographs alone and then reinterpreted them referencing the CAD output. Suspected nodules were enclosed with a bounding box. These bounding boxes were judged correct if there was significant overlap with the ground truth, specifically, if the intersection over union was 0.3 or higher. The sensitivity, specificity, accuracy, PPV, and NPV of the readers' assessments were calculated.
\end{abstract}

Results: In total, 312 chest radiographs were collected as a test dataset, including 59 malignant images (59 nodules of lung cancer) and 253 normal images. The model provided a modest boost to the reader's sensitivity, particularly helping general physicians. The performance of general physicians was improved from 0.47 to 0.60 for sensitivity, from 0.96 to 0.97 for specificity, from 0.87 to 0.90 for accuracy, from 0.75 to 0.82 for PPV, and from 0.89 to 0.91 for NPV while the performance of radiologists was improved from 0.51 to 0.60 for sensitivity, from 0.96 to 0.96 for specificity, from 0.87 to 0.90 for accuracy, from 0.76 to 0.80 for PPV, and from 0.89 to 0.91 for NPV. The overall increase in the ratios of sensitivity, specificity, accuracy, PPV, and NPV were $1.22(1.14-1.30), 1.00(1.00-1.01), 1.03$ $(1.02-1.04), 1.07(1.03-1.11)$, and $1.02(1.01-1.03)$ by using the CAD, respectively.

\footnotetext{
*Correspondence: ai.labo.ocu@gmail.com

'Department of Diagnostic and Interventional Radiology, Graduate School of Medicine, Osaka City University, Osaka, Japan

Full list of author information is available at the end of the article
}

(c) The Author(s). 2021 Open Access This article is licensed under a Creative Commons Attribution 4.0 International License, which permits use, sharing, adaptation, distribution and reproduction in any medium or format, as long as you give appropriate credit to the original author(s) and the source, provide a link to the Creative Commons licence, and indicate if changes were made. The images or other third party material in this article are included in the article's Creative Commons licence, unless indicated otherwise in a credit line to the material. If material is not included in the article's Creative Commons licence and your intended use is not permitted by statutory regulation or exceeds the permitted use, you will need to obtain permission directly from the copyright holder. To view a copy of this licence, visit http://creativecommons.org/licenses/by/4.0/. The Creative Commons Public Domain Dedication waiver (http://creativecommons.org/publicdomain/zero/1.0/) applies to the data made available in this article, unless otherwise stated in a credit line to the data. 
Conclusion: The Al-based CAD was able to improve the ability of physicians to detect nodules of lung cancer in chest radiographs. The use of a CAD model can indicate regions physicians may have overlooked during their initial assessment.

Keywords: Model validation, Chest radiography, Lung Cancer, Artificial intelligence, Deep learning, Computerassisted detection

\section{Background}

Chest radiography is one of the most basic imaging tests in medicine and is the most common examination in routine clinical work such as screening for chest disease, diagnostic workup, and observation. One of the features physicians look for in these chest radiographs is nodules-an indicator of lung cancer, which has the highest mortality rate in the world [1]. In practice, low-dose CT is recommended [2] for lung cancer screening for at-risk individuals rather than chest radiography despite a falsepositive rate of approximately $27 \%$ [2, 3]. Several studies concluded that low-dose CT was superior to radiographs which had a sensitivity of 36-84\% [4-7], varying widely according to tumour size, study population, and reader performance. Other studies showed that 19-26\% of lung cancers visible on chest radiographs were actually missed at the time of initial reading $[6,8]$. However, chest radiography remains the primary diagnostic imaging test for chest conditions because of its advantages over chest CT, including ease of access, lower cost, and lower radiation exposure. Notably, the higher number of chest radiographs per capita than chest $\mathrm{CT}$ indicates that chest radiography has more opportunities to detect lung abnormalities in individuals who are not considered at risk, leading to a diagnostic chest CT.

Since the first computer-assisted detection (CAD) technique for chest radiography was reported in 1988 [9], there have been various developments designed to improve physicians' performance [10-14]. Recently, the application of deep learning (DL), a field of artificial intelligence (AI) $[13,15]$, has led to dramatic, state-ofthe-art improvements in visual object recognition and detection. Automated feature extraction, a critical component of DL, has great potential for application in the medical field [16], especially in radiology [17]. CADs using DL have routinely surpassed the performance of traditional methods. There were two studies which showed that a DL-based CAD may increase physicians' sensitivity for lung cancer detection from chest radiography $[18,19]$. However, these studies only compared the performance of radiologists. The American College of Radiology recommends that radiologists report on all diagnostic imaging [20], but there is a significant shortage of radiologists $[21,22]$. In their absence, general physicians must interpret radiographs themselves.
Patient safety can be improved either by improving the diagnostic accuracy of these physicians or by implementing systems that ensure that initial misinterpretations are corrected before they adversely affect patient care [23]. There are multiple causes of error in interpretating radiographs, but the most common one is recognition error. In other words, it refers to the inability to recognize an anomaly. Moreover, lung cancer was cited as the sixth most common cause for medicolegal action against physicians. The majority of the actions regarding missed lung cancer involved chest radiographs (90\%) [24]. Thus, reading chest radiographs is important for general physicians, however there were no studies evaluating if an AI-based CAD could support not only radiologists, but also general physicians.

The purpose of the present study was to validate a commercially available AI-based CAD that achieved higher performance in detecting lung cancer from chest radiographs. To investigate the ability of this CAD as a support tool, we conducted a multi-vendor, retrospective reader performance test comparing both radiologist and general physicians' performance before and after using the CAD.

\section{Methods}

Study design

A multi-vendor, retrospective clinical validation study comparing the performance of physicians before and after using the CAD was conducted to evaluate the capability of the CAD to assist physicians in detecting lung cancers on chest radiographs. Readers of varying experience level and specialization were included to determine if use of this model on regularly collected radiographs could benefit general physicians. This CAD is commercially available in Japan. The Osaka City University Ethics Board reviewed and approved the protocol of the present study. Since the chest radiographs used in the study had been acquired during daily clinical practice, the need for informed consent was waived by the ethics board. We have created this article in compliance with the STARD checklist [25].

\section{Datasets}

To evaluate the AI-based CAD, chest radiographs of posterior-anterior view were retrospectively collected. 
Chest radiographs with lung cancers were consecutively collected from patients who had been subsequently surgically diagnosed with lung cancer between July 2017 and June 2018 at Osaka City University Hospital, which provides secondary care. The corresponding chest CT, taken within 14 days of the radiograph, were also collected. Chest radiographs with no findings were consecutively collected from patients who reported no nodule/mass finding by chest CT taken within 14 days at the same hospital. Detailed criteria are shown in Additional_File_1. Since the study included patients who visited our institution for the first time, there was no patient overlap among the datasets. Radiographs were taken using a DR CALNEO C 1417 Wireless SQ (Fujifilm Medical), DR AeroDR1717 (Konica Minolta), or DigitalDiagnost VR (Philips Medical Systems).

\section{Eligibility criteria and ground truth labelling}

The eligibility criteria for the radiographs were as follows: (1) Mass lesions larger than $30 \mathrm{~mm}$ in size were excluded. (2) Metastatic lung cancer that was not primary to the lung was excluded. (3) Lung cancers showing anything other than nodular lesions on radiograph were excluded. (4) Nodules in the chest radiographs were annotated with bounding box, referring to chest CT images by two board-certificated radiologists, who had six years (D.U.) and five years (A.S.) of experience interpreting chest radiographs. Ground glass nodules with a diameter of less than $5 \mathrm{~mm}$ were excluded even if they were visible on $\mathrm{CT}$, as they are not considered visible on chest radiographs. When there was disagreement between the annotating radiologists, consensus was achieved by discussion. Chest radiographs with lung cancer presenting nodules, their bounding boxes, and normal chest radiographs were combined to form a test dataset.

\section{The artificial intelligence-based computer-assisted detection model}

The AI-based CAD used in this study is EIRL Chest X-ray Lung nodule (LPIXEL Inc.), commercially available in Japan as of August 2020 as a screening device to find primary lung cancer. The CAD was developed based on an encoder-decoder network categorizing segmentation technique in DL. The CAD was configured to display bounding boxes on all areas of suspected cancer in a radiograph. In the process of internal CAD, the areas suspected of being cancer on chest radiograph were segmented, and the maximum horizontal and vertical diameters of the segmented area are displayed as a bounding box.

\section{Reader performance test}

To evaluate the capability of the CAD to assist physicians, a reader performance test comparing physician performance before and after use of the CAD was conducted. This CAD is certified as a medical software for use by physicians as a second opinion. In other words, physicians first read a chest radiograph without CAD, and then check the CAD output to make a final diagnosis. A total of eighteen readers (nine general physicians and nine radiologists from nine medical institutions) each interpreted the test dataset. The readers had not previously interpreted the same radiographs, did not know the ratio of malignant to normal cases, and clinical information regarding the radiographs was not made available to them. This process was double blinded for the examiners and the reading physicians.

The study protocol was as follows: (1) Each reader was individually trained with 30 radiographs outside the test dataset to familiarize them with the evaluation criteria and use of the CAD. (2) The readers interpreted the radiographs without using the AI-based CAD. If the reader concluded that there was a nodule in the image, then the lesion was annotated with a bounding box on the radiograph. Because the model was designed to produce bounding boxes on all areas that are considered to be positive, we instructed the readers to provide as many bounding boxes as they deemed necessary. (3) The CAD was then applied to the radiograph. (4) The reader interpreted the radiograph again, referring to the output of the CAD. If the reader changed their opinion, he or she annotated again or deleted the previous annotation. (5) The boxes annotated by the reader before and after use of the AI-based CAD were judged correct if the overlap, measured by the intersection over union (IoU), was 0.3 or higher. This value was chosen to meet a stricter standard based on the results from previous studies (Supplementary methods in Additional_File_1).

\section{Statistical analysis}

To evaluate the case-based performance of the readers and the CAD, the accuracy, sensitivity, specificity, positive predictive value (PPV), and negative predictive value (NPV) were evaluated. A lung cancer patient with annotations with an IoU greater than or equal to 0.3 for a ground-truth lesion on a chest radiograph was defined as a true positive (TP) case, a lung cancer patient with annotations with an IoU less than 0.3 for a ground-truth lesion on a chest radiograph was defined as a false negative (FN) case, a non-lung cancer case with no annotations on a chest radiograph was defined as a true negative (TN) case, and a non-lung cancer case with one or more annotations on the chest radiograph was defined as a false positive (FP) case.

To evaluate the lesion-based performance of the readers and the $C A D$, we also determined the mean false positive indications per image (mFPI). The mFPI was defined as the value of the total false positive (FP) lesions 
divided by the total number of images. Annotated lesions were defined as FP if they had an IoU less than 0.3 with a ground-truth lesion. All annotations on a chest radiograph without lung cancer were defined as FP lesions.

These definitions are visually represented in Additional_File_2. In order to assess the improvement of readers' performance metrics for detection of lung nodules due to the CAD, we determined the metrics for cases with and without CAD using Generalized Estimating Equations [26-28]. For each prediction metric, the performance with the CAD was divided by the performance without the CAD to assess the improved ratio. The statistical inferences were performed with two-sided 5\% significance level. Decisions of readers before and after referencing $C A D$ output were counted to evaluate the CAD effect. Two of the authors (D.U. and D.K.) performed all analyses using $\mathrm{R}$, version 3.6.0.

\section{Results \\ Datasets}

From July 2017 through June 2018, we consecutively collected 122 chest radiographs from lung cancer patients. Eight radiographs were excluded because they contained metastases, 44 radiographs were excluded because the nodules were more than $30 \mathrm{~mm}$ in size, and four radiographs were excluded because the lesion showing was not nodular. The 66 remaining radiographs were annotated by author radiologists and seven radiographs were subsequently excluded because they concluded that the nodule was not visible on the chest radiograph. Thus, 59 radiographs from 59 patients were used as the malignant set. From July 2017 through June 2018, we collected 253 chest radiographs from patients with no nodule/mass finding via CT within 14 days. A total of 312 radiographs (59 malignant radiographs from 59 patients and 253 non-malignant radiographs from 253 patients; age range, 33-92 years; mean age \pm standard deviation, $59 \pm 13$ years) were used for the test dataset to examine reader performance.

A flowchart of the eligibility criteria for the dataset is shown in Additional_File_3. Detailed demographic information of the test dataset is provided in Table 1.

\section{The deep learning-based computer-assisted detection model performance}

The standalone CAD sensitivity, specificity, accuracy, PPV, and NPV were 0.66 (0.53-0.78), 0.96 (0.92-0.98), 0.90 (0.86-0.93), $0.78(0.64-0.88)$, and $0.92(0.88-0.95)$ with mFPI of 0.05 , respectively.

\section{Reader performance test}

The demographic information of the readers is provided in Supplementary Table 1 in Additional_File_1. All
Table 1 Dataset demographics

\begin{tabular}{|c|c|}
\hline & Test dataset \\
\hline Male & 153 \\
\hline Female & 159 \\
\hline Mean age \pm standard deviation (years \pm SD) & $61.6 \pm 11.4$ \\
\hline Total no. of radiographs & 312 \\
\hline Total no. of cancers & 59 \\
\hline \multicolumn{2}{|l|}{ Size } \\
\hline Average \pm SD [mm] & $17.9 \pm 6.28$ \\
\hline$\leqq 10 \mathrm{~mm}$ & $7(12 \%)$ \\
\hline $11-20 \mathrm{~mm}$ & $33(56 \%)$ \\
\hline $21-30 \mathrm{~mm}$ & $19(32 \%)$ \\
\hline \multicolumn{2}{|l|}{ Laterality } \\
\hline Right & 39 (66\%) \\
\hline Left & $20(34 \%)$ \\
\hline \multicolumn{2}{|l|}{ Location } \\
\hline Upper & $23(39 \%)$ \\
\hline Middle & $31(53 \%)$ \\
\hline Lower & $5(8 \%)$ \\
\hline \multicolumn{2}{|l|}{ Overlap } \\
\hline Heart & $2(3 \%)$ \\
\hline Clavicle & $6(10 \%)$ \\
\hline Diaphragm & $1(2 \%)$ \\
\hline Hilar vessels & $3(5 \%)$ \\
\hline \multicolumn{2}{|l|}{ Manufacturer (\% radiographs with cancer) } \\
\hline FUJIFILM & $6 / 89(7 \%)$ \\
\hline KONICA & $31 / 113(27 \%)$ \\
\hline Philips & $22 / 110(20 \%)$ \\
\hline
\end{tabular}

Data are $\mathrm{n}$ unless otherwise noted. SD: standard deviation

readers improved their overall performance by referring to the CAD output. The overall increases for reader performance due to using the CAD for sensitivity, specificity, accuracy, PPV, and NPV were 1.22 (1.14-1.30), 1.00 (1.00-1.01), 1.03 (1.02-1.04), 1.07 (1.03-1.11), and 1.02 (1.01-1.03), respectively (Table 2 ). General physicians benefited more from the use of the CAD than radiologists did. The performance of general physicians was improved from 0.47 to 0.60 for sensitivity, from 0.96 to 0.97 for specificity, from 0.87 to 0.90 for accuracy, from 0.75 to 0.82 for PPV, and from 0.89 to 0.91 for NPV while the performance of radiologists was improved from 0.51 to 0.60 for sensitivity, from 0.96 to 0.96 for specificity, from 0.87 to 0.90 for accuracy, from 0.76 to 0.80 for PPV, and from 0.89 to 0.91 for NPV. Detailed results per reader are in Supplementary Table 2 in Additional_File_1. The sensitivity of readers before and after using the CAD is shown as a bilinear graph in Fig. 1. The rate of improvement was particularly high for 
Table 2 Results of readers with and without CAD

\begin{tabular}{|c|c|c|c|c|c|c|c|c|c|c|}
\hline & \multicolumn{2}{|c|}{ General physicians } & \multicolumn{2}{|c|}{ Radiologists } & \multicolumn{2}{|l|}{ Overall } & \multirow{2}{*}{$\begin{array}{l}\text { Ratio } \\
\text { (CAD/Non-CAD) }\end{array}$} & \multicolumn{2}{|c|}{ 95\% Confidence Interval } & \multirow[b]{2}{*}{$P$ valu } \\
\hline & Non-CAD & CAD & Non-CAD & CAD & Non-CAD & CAD & & Lower & Upper & \\
\hline Sensitivity & 0.47 & 0.60 & 0.51 & 0.60 & 0.49 & 0.60 & 1.22 & 1.14 & 1.30 & $<0.001$ \\
\hline Specificity & 0.96 & 0.97 & 0.96 & 0.96 & 0.96 & 0.97 & 1.00 & 1.00 & 1.01 & 0.221 \\
\hline Accuracy & 0.87 & 0.90 & 0.87 & 0.90 & 0.87 & 0.90 & 1.03 & 1.02 & 1.04 & $<0.001$ \\
\hline Positive Predictive Value & 0.75 & 0.82 & 0.76 & 0.80 & 0.75 & 0.81 & 1.07 & 1.03 & 1.11 & 0.002 \\
\hline Negative Predictive Value & 0.89 & 0.91 & 0.89 & 0.91 & 0.89 & 0.91 & 1.02 & 1.01 & 1.03 & $<0.001$ \\
\hline
\end{tabular}

CAD: computer-assisted detection

general physicians (Fig. 2). General physicians were more likely to change their assessment from FN to TP by referencing correct positive CAD output (68 times (0.59) in general physicians, 49 (0.49) in radiologists) and from FP to TN by correct negative CAD output (29 times (0.36) in general physicians, 24 times $(0.29)$ in radiologists) (Table 3 ). The less experienced the reader was, the higher the rate of sensitivity improvement (Fig. 3). Conversely, the more experienced the readers were, the more limited the support capabilities of the CAD were. Radiologists were less likely to change their opinion than general physicians, and it was more difficult for radiologists to change their decisions from FP to TN (24 times) than from FN to TP (49 times). Results for readers' determinations on TP radiographs were also calculated (Supplementary Table 3 in Additional_File_1). Additional_File_4 shows an instance in which a physician mistakenly changed their decision from TP to FN due to the FN output of the CAD. Instances in which physicians correctly changed their decision from $\mathrm{FN}$ to TP due to the TP output of the CAD can be seen in Fig. 3 and Additional_File_5.

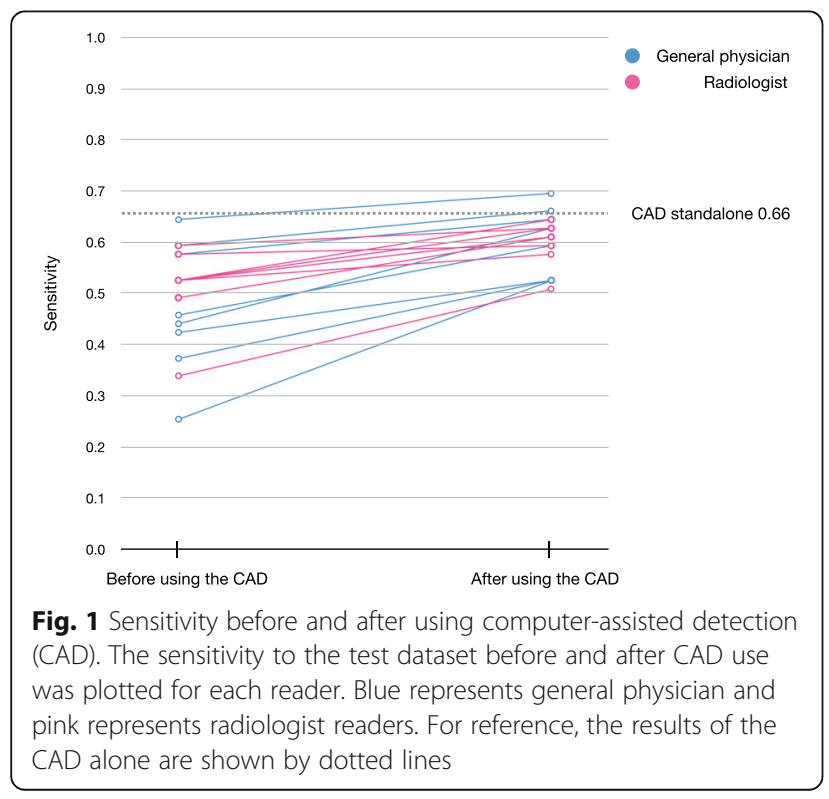

\section{Discussion}

We performed a multi-vendor, retrospective clinical validation to compare the performance of readers before and after using an AI-based CAD. The number of TPs that could be detected in the test dataset was greater than that of any human readers alone. The results of the present study indicate that the AI-based CAD can improve physician performance. Additionally, general physicians benefited more from the use of the CAD than radiologists did.

This is the first study to evaluate the performance not only of radiologists but also general physicians in their evaluation of chest radiographs with AI-based CAD assistance. A chest radiograph is one of the most basic tests that every physician is expected to be able to interpret to some extent, yet detection of pulmonary nodules on chest radiographs is prone to errors. Previous studies have found that about $20 \%$ of lung cancers visible on chest radiographs were actually missed at the time of

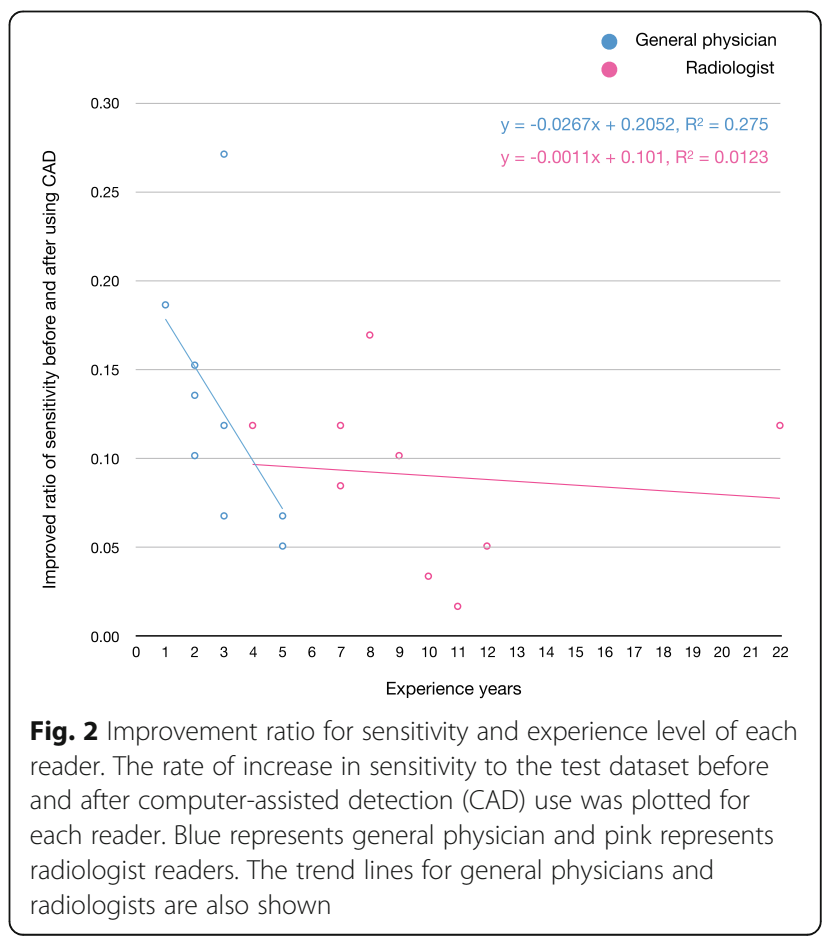


Table 3 Decisions in readers before and after referencing CAD output

\begin{tabular}{|c|c|c|c|c|c|c|c|c|}
\hline \multirow{2}{*}{$\frac{\text { CAD results }}{\text { Reader results }}$} & \multicolumn{2}{|c|}{ TP } & \multicolumn{2}{|c|}{ TN } & \multicolumn{2}{|c|}{$\mathrm{FN}$} & \multicolumn{2}{|c|}{ FP } \\
\hline & & & & & & & & \\
\hline Before CAD use & \multicolumn{2}{|c|}{ FN } & \multicolumn{2}{|c|}{$\mathrm{FP}$} & \multicolumn{2}{|c|}{$\mathrm{TP}$} & \multicolumn{2}{|c|}{ TN } \\
\hline After CAD use & $\mathrm{TP}$ & $\mathrm{FN}$ & TN & $\mathrm{FP}$ & $\mathrm{FN}$ & TP & FP & $\mathrm{TN}$ \\
\hline \multicolumn{9}{|l|}{ General physicians } \\
\hline Reader 1 & 11 & 3 & 2 & 5 & 0 & 1 & 3 & 8 \\
\hline Reader 2 & 8 & 6 & 5 & 9 & 0 & 2 & 1 & 10 \\
\hline Reader 3 & 9 & 9 & 0 & 2 & 0 & 1 & 0 & 11 \\
\hline Reader 4 & 6 & 11 & 1 & 9 & 0 & 3 & 0 & 11 \\
\hline Reader 5 & 7 & 4 & 4 & 0 & 0 & 1 & 2 & 9 \\
\hline Reader 6 & 16 & 8 & 13 & 3 & 0 & 0 & 6 & 4 \\
\hline Reader 7 & 4 & 2 & 0 & 18 & 0 & 2 & 0 & 9 \\
\hline Reader 8 & 3 & 0 & 3 & 1 & 0 & 2 & 1 & 9 \\
\hline Reader 9 & 4 & 5 & 1 & 5 & 0 & 4 & 1 & 10 \\
\hline All general physicians & 68 & 48 & 29 & 52 & 0 & 16 & 14 & 81 \\
\hline \multicolumn{9}{|l|}{ Radiologists } \\
\hline Reader 10 & 7 & 4 & 2 & 13 & 0 & 1 & 4 & 7 \\
\hline Reader 11 & 5 & 4 & 0 & 6 & 0 & 1 & 0 & 11 \\
\hline Reader 12 & 7 & 5 & 2 & 0 & 0 & 2 & 2 & 9 \\
\hline Reader 13 & 11 & 10 & 11 & 2 & 1 & 1 & 2 & 9 \\
\hline Reader 14 & 6 & 4 & 0 & 13 & 0 & 2 & 7 & 3 \\
\hline Reader 15 & 2 & 6 & 5 & 7 & 0 & 4 & 2 & 8 \\
\hline Reader 16 & 1 & 8 & 0 & 2 & 0 & 4 & 0 & 11 \\
\hline Reader 17 & 3 & 8 & 0 & 10 & 0 & 3 & 0 & 11 \\
\hline Reader 18 & 7 & 3 & 4 & 5 & 0 & 2 & 4 & 5 \\
\hline All radiologists & 49 & 52 & 24 & 58 & 1 & 20 & 21 & 74 \\
\hline
\end{tabular}

Data are n. CAD: computer-assisted detection, FN: false negative, TP: true positive, FP: false positive, TN: true negative initial reading $[6,8]$. Physicians are aware of the risks misreading can cause, such as patient harm or medicolegal action, thus, the task can be difficult and distressing for inexperienced or general physicians. For this reason, we asked less experienced physicians to participate in this study to measure how much their performance could be improved with CAD support. Our results show that using this model could support both general physicians and radiologists in the detection of lung nodules.

The CAD increased physicians' sensitivity with statistical significance without increasing the number of false positives. This is due to the high sensitivity of the CAD. The standalone CAD performance included a sensitivity of $0.66(0.53-0.78)$ with mFPI of 0.05 . This was comparable to or better than all of the individual physicians' performance in our study. Since most AI models are designed to prevent misses, the trade-off is generally an increase in the number of false positives. These false positives can lead to an increase in unnecessary testing $[29,30]$. This study indicates that more lung cancers could be detected without the need for chest CT or biopsy after implementation of this model into a chest radiography viewer.

To compare our results to previous CAD studies, this CAD shows a considerably lower mFPI. Previous studies showed an mFPI of 0.9-3.9 [18, 19, 31-37], while ours was 0.05 . There are two studies $[18,19]$ with particularly high sensitivity and low mFPI. Sim et al. [19] showed a CAD sensitivity of 0.67 and an mFPI of 0.2 , but their dataset excluded nodules smaller than $10 \mathrm{~mm}$. Nam et al. [18] showed a CAD sensitivity of $0.69-0.82$ and mFPI of 0.02-0.34, but their datasets contained a high percentage of masses greater than $30 \mathrm{~mm}$ and the nodules were not pathologically proven to be malignant. One possible reason why the CAD used in our study

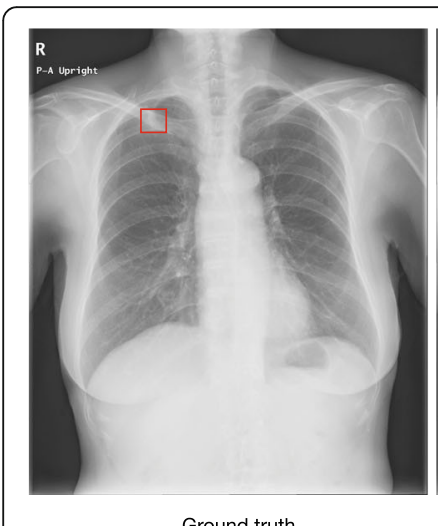

Ground truth

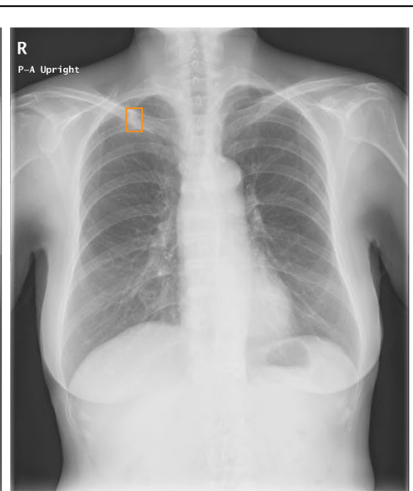

CAD results

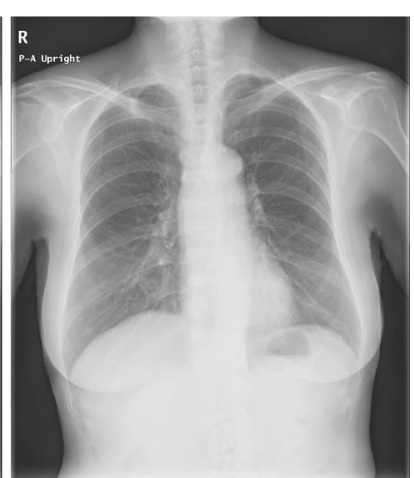

Reader's result before using the CAD

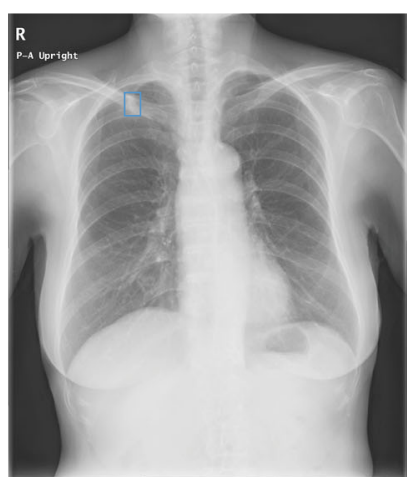

Reader's result after using the CAD

Fig. 3 Example of a case in which physician correctly changed their decision due to computer-assisted detection (CAD) output. A case involving a 70-year-old woman with a nodule in the right upper pulmonary field overlapping the clavicle changed from false negative to true positive by a general physician with three years of experience (Reader 5), by referring to the true positive results of the CAD 
achieved high sensitivity with low mFPI was that it was created with a segmentation-based deep learning model, unlike other studies. Segmentation, also known as pixel labelling, deals with pixel-by-pixel information, which allows us to extract lesions more finely than general classification and detection models. The datasets in the former studies do not resemble a typical screening cohort. The sensitivity of the CAD in this study was found to be 0.66 with $0.05 \mathrm{mFPI}$. Although CAD has been applied to many fields, the typical increase in false positives remains a problem. This model was able to increase the sensitivity for true malignancies while reducing the number of false positives presented.

The advantage of using the AI model to the general physician was higher than that to the radiologist. In cases where the reader made a mistake (FN or FP) and the CAD showed the correct output (TP or TN), the general physicians were more likely to correct their error than the radiologists. Additionally, radiologists changed TN to FP more often (21 cases, or $22 \%)$ than general physicians (14 cases, or $15 \%$ ) when the CAD presented FP output. The results showed that general physicians benefit more from this CAD than radiologists.

The limitations of this study include that the test dataset was collected from a single institution, although the readers who participated were from multiple institutions. The weakness of the CAD in detecting nodules of less than $10 \mathrm{~mm}$ may also be a limiting factor. The CAD could identify only one of the seven nodules under $10 \mathrm{~mm}$, while most readers did not identify even one nodule. If the performance of CAD is improved, there is a possibility of detecting lung cancer at an earlier stage. Our dataset did not have radiographs with multiple lesions. In actual screening, single lesions are most common, but multiple lesions may be present.

\section{Conclusions}

We conducted a multi-vendor, retrospective clinical validation to compare the performance of readers before and after using a commercially available AI-based CAD. The AI-based CAD supported physicians in the detection of lung cancers in chest radiography. We hope that the correct use of CAD in chest radiography, a basic and ubiquitous clinical examination, will lead to better medical care by preventing false negative assessments and supporting physicians' determinations.

\section{Abbreviations}

Al: Artificial intelligence; CAD: Computer-assisted detection; DL: Deep learning; FN: False negative; FP: False positive; loU: Intersection over union; mFPI: Mean false positive indications per image; NPV: Negative predictive value; PPV: Positive predictive value; TN: True negative; TP: True positive

\section{Supplementary Information}

The online version contains supplementary material available at https://doi. org/10.1186/s12885-021-08847-9.

Additional File 1. Supplementary Methods, Comments, Tables, and Supplementary Figure Legends

Additional File 2. Supplementary Fig. 1. Metric definitions for cases and lesions

Additional File 3. Supplementary Fig. 2. Eligibility of chest radiographs for test dataset

Additional File 4. Supplementary Fig. 3. Example of a case in which a physician mistakenly changed their decision from true positive to false negative due to the false negative output of the CAD

Additional File 5. Supplementary Fig. 4. Other examples of cases in which physicians correctly changed their decision from false negative to true positive due to the true positive output of the CAD

\section{Acknowledgements}

We thank LPIXEL Inc. for their collaboration.

\section{Authors' contributions}

DU managed the study, performed the analysis, and prepared the manuscript. DK confirmed the analysis. AY designed the study and reviewed the manuscript. AS, TM, reviewed the manuscript. SLW revised and proofread the manuscript. $\mathrm{NI}, \mathrm{T}, \mathrm{HK}, \mathrm{HI}$ prepared the data and reviewed the manuscript. NN and YM supervised this study.

\section{Funding}

There was no funding for this study.

\section{Availability of data and materials}

The datasets used and/or analysed during the current study are available from the corresponding author on reasonable request. The commercial software used in this study is available from LPIXEL at https://eirl.ai/eirlchest_nodule.

\section{Declarations}

Ethics approval and consent to participate

Administrative permissions from Osaka City University Ethics Board were obtained to access the raw data. The Osaka City University Ethics Board reviewed and approved the protocol of the present study. Since the chest radiographs used in the study had been acquired during daily clinical practice, the need for informed consent was waived by the ethics board. Osaka City University Hospital accepted the use of the raw data based on the results of the ethics board, under compliance with the hospital's anonymization regulations.

Consent for publication Not applicable.

\section{Competing interests}

The authors report no conflicts of interest.

\section{Author details}

${ }^{1}$ Department of Diagnostic and Interventional Radiology, Graduate School of Medicine, Osaka City University, Osaka, Japan. ²Department of Surgery,

Graduate School of Medicine, Osaka City University, Osaka, Japan.

${ }^{3}$ Department of Medical Statistics, Graduate School of Medicine, Osaka City University, Osaka, Japan.

Received: 28 June 2021 Accepted: 7 October 2021

Published online: 18 October 2021

\section{References}

1. Bray F, Ferlay J, Soerjomataram I, Siegel RL, Torre LA, Jemal A. Global cancer statistics 2018: GLOBOCAN estimates of incidence and mortality worldwide for 36 cancers in 185 countries. CA Cancer J Clin. 2018:68(6):394-424. https://doi.org/10.3322/caac.21492. 
2. Manser R, Lethaby A, Irving LB, Stone C, Byrnes G, Abramson MJ, et al. Screening for lung cancer. Cochrane Database of Systematic Reviews. 2013; 2013:Cd001991.

3. Team NLSTR, Aberle DR, Adams AM, Berg CD, Black WC, Clapp JD, et al. Reduced lung-cancer mortality with low-dose computed tomographic screening. N Engl J Med. 2011;365(5):395-409. https://doi.org/10.1056/ NEJMoa1102873.

4. Aberle DR, DeMello S, Berg CD, Black WC, Brewer B, Church TR, et al. Results of the two incidence screenings in the National Lung Screening Trial. N Engl J Med. 2013;369(10):920-31. https://doi.org/10.1056/NEJMoa1208962.

5. de Hoop B, Schaefer-Prokop C, Gietema HA, de Jong PA, van Ginneken B, van Klaveren RJ, et al. Screening for lung cancer with digital chest radiography: sensitivity and number of secondary work-up CT examinations. Radiology. 2010;255(2):629-37. https://doi.org/10.1148/radiol.09091308.

6. Gavelli G, Giampalma E. Sensitivity and specificity of chest X-ray screening for lung cancer: review article. Cancer. 2000;89(S11):2453-6. https://doi.org/1 0.1002/1097-0142(20001201)89:11+<2453::AID-CNCR21>3.0.CO;2-M.

7. Potchen EJ, Cooper TG, Sierra AE, Aben GR, Potchen MJ, Potter MG, et al. Measuring performance in chest radiography. Radiology. 2000;217(2):456-9. https://doi.org/10.1148/radiology.217.2.ro0nv14456.

8. Quekel LG, Kessels AG, Goei R, van Engelshoven JM. Miss rate of lung cancer on the chest radiograph in clinical practice. Chest. 1999;115(3):720-4. https://doi.org/10.1378/chest.115.3.720.

9. Giger ML, Doi K, MacMahon H. Image feature analysis and computer-aided diagnosis in digital radiography. III. Automated detection of nodules in peripheral lung fields. Med Phys. 1988;15(2):158-66. https://doi.org/10.111 8/1.596247.

10. van Ginneken B, ter Haar Romeny BM, Viergever MA. Computer-aided diagnosis in chest radiography: a survey. IEEE Trans Med Imaging. 2001; 20(12):1228-41. https://doi.org/10.1109/42.974918.

11. Shiraishi J, Li Q, Appelbaum D, Doi K. Computer-aided diagnosis and artificial intelligence in clinical imaging. Semin Nucl Med. 2011;41(6):449-62. https://doi.org/10.1053/j.semnuclmed.2011.06.004.

12. Qin C, Yao D, Shi Y, Song Z. Computer-aided detection in chest radiography based on artificial intelligence: a survey. Biomed Eng Online. 2018;17(1):113. https://doi.org/10.1186/s12938-018-0544-y.

13. Yang Y, Feng X, Chi W, Li Z, Duan W, Liu H, et al. Deep learning aided decision support for pulmonary nodules diagnosing: a review. J Thorac Dis. 2018;2018:S867-75. https://doi.org/10.21037/jtd.2018.02.57.

14. Lee SM, Seo JB, Yun J, Cho Y, Vogel-Claussen J, Schiebler ML, et al. Deep learning applications in chest radiography and computed tomography: current state of the art. J Thorac Imaging. 2019;34(2):75-85. https://doi.org/1 0.1097/RTI.0000000000000387.

15. LeCun Y, Bengio Y, Hinton G. Deep learning. Nature. 2015;521(7553):436-44. https://doi.org/10.1038/nature14539.

16. Hinton G. Deep learning - a technology with the potential to transform health care. JAMA. 2018;320(11):1101-2. https://doi.org/10.1001/jama.201 8.11100 .

17. Ueda D, Shimazaki A, Miki Y. Technical and clinical overview of deep learning in radiology. Jpn J Radiol. 2019;37(1):15-33. https://doi.org/10.1007/ s11604-018-0795-3.

18. Nam JG, Park S, Hwang EJ, Lee JH, Jin K, Lim KY, et al. Development and validation of deep learning-based automatic detection algorithm for malignant pulmonary nodules on chest radiographs. Radiology. 2019;290(1): 218-28. https://doi.org/10.1148/radiol.2018180237.

19. Sim Y, Chung MJ, Kotter E, Yune S, Kim M, Do S, et al. Deep convolutional neural network-based software improves radiologist detection of malignant lung nodules on chest radiographs. Radiology. 2020;294(1):199-209. https:// doi.org/10.1148/radiol.2019182465.

20. American College of Radiology. ACR standard for general radiography. In: ACR-SPR Practice Parameter For General Radiography. American College of Radiology. 2000. https://www.acr.org/-/media/ACR/Files/Practice-Para meters/RadGen.pdf. Accessed 15 Aug 2021.

21. Bender CE, Bansal S, Wolfman D, Parikh JR. 2018 ACR Commission on human resources workforce survey. J am Coll Radiol. 2019;16(4 Pt a):508-12. doi: https://doi.org/10.1016/j.jacr.2018.12.034. PMID: 30745040, 16, 508, 512.

22. The Royal College of Radiologists. In: Clinical Radiology U.K. Workforce Census Report 2018. The Royal College of Radiologists. 2019. https://www. rcr.ac.uk/system/files/publication/field_publication_files/clinical-radiology-ukworkforce-census-report-2018.pdf. (Accessed 15 Aug 2021).
23. Kripalani S, Williams MV, Rask K. Reducing errors in the interpretation of plain radiographs and computed tomography scans. In. 2001;2001.

24. Fardanesh $\mathrm{M}$, White $\mathrm{C}$. Missed lung cancer on chest radiography and computed tomography. Semin Ultrasound CT MR. 2012 Aug;33(4):280-7. 22 824118. https://doi.org/10.1053/j.sult.2012.01.006.

25. Bossuyt PM, Reitsma JB, Bruns DE, Gatsonis CA, Glasziou PP, Irwig L, et al. STARD 2015: an updated list of essential items for reporting diagnostic accuracy studies. BMJ. 2015;351:h5527. https://doi.org/10.1136/bmj.h5527.

26. Liang KY, Zeger SL. Longitudinal data analysis using generalized linear models. Biometrika. 1986;73(1):13-22. https://doi.org/10.1093/biomet/73.1.13.

27. Zeger SL, Liang KY. The analysis of discrete and continuous longitudinal data. Biometrics. 1986;42(1):121-30. https://doi.org/10.2307/2531248.

28. Kosinski AS. A weighted generalized score statistic for comparison of predictive values of diagnostic tests. Statist Med. 2013;32(6):964-77. https:// doi.org/10.1002/sim.5587.

29. Haber M, Drake A, Nightingale J. Is there an advantage to using computer aided detection for the early detection of pulmonary nodules within chest X-ray imaging? Radiography (Lond). 2020 Aug;26(3):e170-8. https://doi.org/1 0.1016/j.radi.2020.01.002

30. Qin C, Yao D, Shi Y, Song Z. Computer-aided detection in chest radiography based on artificial intelligence: a survey. Biomed Eng Online. 2018 Aug 22; 17(1):113. https://doi.org/10.1186/s12938-018-0544-y.

31. De Boo DW, Uffmann M, Weber M, et al. Computer-aided detection of smal pulmonary nodules in chest radiographs: an observer study. Acad Radiol. 2011;18(12):1507-14. https://doi.org/10.1016/j.acra.2011.08.008.

32. de Hoop B, De Boo DW, Gietema HA, et al. Computer-aided detection of lung cancer on chest radiographs: effect on observer performance. Radiology. 2010;257(2):532-40. https://doi.org/10.1148/radiol.10092437.

33. Lee KH, Goo JM, Park CM, Lee HJ, Jin KN. Computer-aided detection of malignant lung nodules on chest radiographs: effect on observers' performance. Korean J Radiol. 2012;13(5):564-71. https://doi.org/10.3348/ kjr.2012.13.5.564.

34. Meziane M, Mazzone P, Novak E, Lieber ML, Lababede O, Phillips M, et al. A comparison of four versions of a computer-aided detection system for pulmonary nodules on chest radiographs. J Thorac Imaging. 2012;27(1):5864. https://doi.org/10.1097/RTI.0b013e3181f240bc.

35. Novak RD, Novak NJ, Gilkeson R, Mansoori B, Aandal GE. A comparison of computer-aided detection (CAD) effectiveness in pulmonary nodule identification using different methods of bone suppression in chest radiographs. J Digit Imaging. 2013;26(4):651-6. https://doi.org/10.1007/s10278-0129565-4.

36. van Beek EJR, Mullan B, Thompson B. Evaluation of a real-time interactive pulmonary nodule analysis system on chest digital radiographic images: a prospective study. Acad Radiol. 2008;15(5):571-5. https://doi.org/10.1016/j.a cra.2008.01.018.

37. $\mathrm{Xu} Y, \mathrm{Ma} \mathrm{D}, \mathrm{He} \mathrm{W}$. Assessing the use of digital radiography and a real-time interactive pulmonary nodule analysis system for large population lung cancer screening. Eur J Radiol. 2012;81(4):e451-6. https://doi.org/10.1016/j. ejrad.2011.04.031.

\section{Publisher's Note}

Springer Nature remains neutral with regard to jurisdictional claims in published maps and institutional affiliations.

Ready to submit your research? Choose BMC and benefit from:

- fast, convenient online submission

- thorough peer review by experienced researchers in your field

- rapid publication on acceptance

- support for research data, including large and complex data types

- gold Open Access which fosters wider collaboration and increased citations

- maximum visibility for your research: over $100 \mathrm{M}$ website views per year

At $\mathrm{BMC}$, research is always in progress.

Learn more biomedcentral.com/submission 